\title{
The Ectopic Expression of a Pectin Methyl Esterase Inhibitor Increases Pectin Methyl Esterification and Limits Fungal Diseases in Wheat
}

\author{
Chiara Volpi, ${ }^{1}$ Michela Janni, ${ }^{1}$ Vincenzo Lionetti, ${ }^{2}$ Daniela Bellincampi, ${ }^{2}$ Francesco Favaron, ${ }^{3}$ and \\ Renato D'Ovidio'
}

${ }^{1}$ Department of Agrobiology and Agrochemistry, University of Tuscia, 01100 Viterbo, Italy; ${ }^{2}$ Department of Biology and Biotechnology "Charles Darwin", University of Rome "Sapienza", 00185 Rome; ${ }^{3}$ Department of Land, Environment, Agriculture and Forestry, University of Padua, Legnaro (PD), Italy.

Submitted 31 January 2011. Accepted 9 May 2011.

\begin{abstract}
Cell wall pectin methyl esterification can influence plant resistance because highly methyl-esterified pectin can be less susceptible to the hydrolysis by pectic enzymes such as fungal endopolygalacturonases (PG). Pectin is secreted into the cell wall in a highly methyl-esterified form and, here, is de-methyl esterified by pectin methyl esterase (PME). The activity of PME is controlled by specific protein inhibitors called PMEI; consequently, an increased inhibition of PME by PMEI might modify the pectin methyl esterification. In order to test the possibility of improving wheat resistance by modifying the methyl esterification of pectin cell wall, we have produced durum wheat transgenic lines expressing the PMEI from Actinidia chinensis (AcPMEI). The expression of AcPMEI endows wheat with a reduced endogenous PME activity, and transgenic lines expressing a high level of the inhibitor showed a significant increase in the degree of methyl esterification. These lines showed a significant reduction of disease symptoms caused by the fungal pathogens Bipolaris sorokiniana or Fusarium graminearum. This increased resistance was related to the impaired ability of these fungal pathogens to grow on methyl-esterified pectin and to a reduced activity of the fungal PG to hydrolyze methyl-esterified pectin. In addition to their importance for wheat improvement, these results highlight the primary role of pectin despite its low content in the wheat cell wall.
\end{abstract}

The plant cell wall represents a main barrier to the colonization of host plant tissue in several plant-pathogen interactions. The complex structure of the plant cell wall is composed mostly of the polysaccharides cellulose, hemicellulose, and pectin. Pectin is composed mainly of homogalacturonan (HG) that consists of a linear homopolymer of 1,4-linked $\alpha$-D-galacturonic acid (GalA) with a degree of polymerization of approximately 100 residues (Mohnen 2008). Pectin is synthesized in the Golgi apparatus, secreted into the cell wall in a highly methyl-esterified form and, here, de-esterified to varying degree in a spatially regulated manner not yet well understood (Mohnen 2008). The level of methyl esterification of cell wall pectin can have a great impact on cell wall structure and prop-

Corresponding author: R. D’Ovidio; E-mail: dovidio@unitus.it

* The $\boldsymbol{e}$-Xtra logo stands for "electronic extra" and indicates that one supplementary table is published online. erties, with consequences on several physiological processes (Willats et al. 2001a; Wolf et al. 2009), including cell adhesion (Willats et al. 2001b), pollen tube growth (Bosch et al. 2005), phyllotaxis (Peaucelle et al. 2008), and cell elongation (Pelletier et al. 2010). The degree and pattern of pectin methyl esterification can also influence plant response to pathogens. In potato, the higher degree of cell wall pectin methyl esterification of stem, tuber cortex, and medullary tissue correlates with resistance against some bacterial Erwinia (now Pectobacterium or Dickeya) species (Marty et al. 1997; McMillan et al. 1993). A higher degree of pectin methylation was also found in tomato genotypes resistant to Pseudomonas syringae pv. tomato (Venkatesh 2002) or Ralstonia solanacearum (Wydra and Beri 2006) compared with the susceptible ones. In bean, isolines resistant and susceptible to the fungal pathogen Colletotrichum lindemuthianum showed a different degree of methyl esterification, with the resistant isoline having the higher level (Boudart et al. 1998).

The methyl esterification of pectin is mainly controlled by pectin methyl esterases (PME) (Willats et al. 2001a). PME activity, which catalyzes the de-methyl esterification of the C6 linked methyl ester group of $\mathrm{HG}$, is tightly regulated by its endogenous inhibitor protein, called pectin methyl esterase inhibitor (PMEI) (Giovane et al. 2004; Pelloux et al. 2007). PMEI is active against plant PME by forming a stoichiometric $1: 1$ complex in which the inhibitor binds the putative active site of the enzyme (Di Matteo et al. 2005). The effectiveness of PMEI in controlling PME activity has been demonstrated also by the overexpression of AtPMEI-1 or AtPMEI-2 in transgenic Arabidopsis plants. Both proteins interacted with endogenous PME isoforms, and transgenic tissues showed a significant reduction of PME activity (Lionetti et al. 2007). These transgenic plants also showed an increased level of cell wall pectin methyl esterification and a significant reduction of symptoms caused by Botrytis cinerea (Lionetti et al. 2007).

Grass species contain a low level of pectin in their cell wall (Vogel 2008); however, differences in methyl ester distribution of HG have been found in near-isogenic lines resistant and susceptible to the fungal pathogen Puccinia graminis f. $\mathrm{sp}$. tritici (Wietholter et al. 2003). In particular, a more random distribution of methyl ester has been detected in isogenic resistant lines compared with a more blockwise distribution found in the susceptible lines (Wietholter et al. 2003). In order to verify whether the increased accumulation of PMEI may increase wheat resistance to fungal pathogens, we have produced and analyzed transgenic durum wheat lines that accumulate 
AcPMEI, a deeply characterized PMEI from Actinidia chinensis (Camardella et al. 2000). We report here that the overexpression of Acpmei in wheat, a grass species with a low level of pectin in the cell wall, significantly improved the plant resistance to the fungal pathogens Bipolaris sorokiniana and Fusarium graminearum, the causal agents of spot blotch and Fusarium head blight (FHB), respectively.

\section{RESULTS}

Production of wheat transgenic plants expressing AcPMEI.

The capability of purified AtPMEI-1, AtPMEI-2 (Raiola et al. 2004), or AcPMEI (Camardella et al. 2000) to inhibit wheat PME activity was verified by radial gel diffusion assay. Total proteins $(9 \mu \mathrm{g})$ extracted from leaves of Triticum durum 'Svevo' were loaded alone or in combination with $15 \mathrm{ng}$ of purified inhibitors. All three inhibitors inhibited wheat PME activity, with AcPMEI showing $100 \%$ inhibition and both AtPMEI exhibiting approximately $75 \%$ inhibition. Based on this result, the Acpmei gene was used to prepare the pUbi::Acpmei cassette to transform wheat plants.

In total, 2,229 T. durum Svevo immature embryos were cotransformed using pUbi::Acpmei and pUbi::bar in two separate bombardment experiments. In all, 139 independent regenerated lines were obtained and analyzed for the presence of the transgene by polymerase chain reaction (PCR) using the specific primers AcpmeiF4/AcpmeiR4. Seventy-one $T_{0}$ independent transgenic plants resistant to the bialaphos herbicide and containing the Acpmei gene were obtained, for a transformation efficiency of $3.4 \%$, which is within the typical efficiency obtained in stable wheat transformation.

In order to verify the inhibitory activity of transgeneencoded AcPMEI, $\mathrm{T}_{1}$ positive lines were analyzed for the endogenous PME activity by radial gel diffusion assay (Lionetti et al. 2007). Total leaf protein extract $(10 \mu \mathrm{g})$ from transgenic and wild-type (WT) plants was loaded onto the agarose gel containing pectin (70 to $75 \%$ methyl esterified) from apple, and the halo produced by the endogenous PME activity was measured. Relative PME activity of transgenic lines with respect to the untransformed WT was calculated. Of $36 \mathrm{~T}_{1}$ lines analyzed, 12 transgenic lines showed PME activity that varied from approximately 5 to $80 \%$ compared with the PME activity of the control plants (Table 1). The Acpmei lines homozygous for the transgene showing a high (MJ15-151 and MJ15-69) and low (MJ15-57) reduction of endogenous PME activity

Table 1. Quantification of endogenous pectin methyl esterase (PME) activity in 12 Acpmei lines and wild-type (WT) plants determined by radial gel diffusion assay ${ }^{\mathrm{Z}}$

\begin{tabular}{lc}
\hline Lines & Relative PME activity $(\%)$ \\
\hline WT & 100 \\
MJ15-151 & $5.2 \pm 0.5$ \\
MJ15-70 & $76.4 \pm 1.6$ \\
MJ15-69 & $5.0 \pm 0.3$ \\
MJ15-61 & $10.0 \pm 1.6$ \\
MJ15-57 & $60.0 \pm 3.5$ \\
MJ15-49 & $10.0 \pm 1.6$ \\
MJ15-93 & $12.8 \pm 1.8$ \\
MJ15-25 & $66.0 \pm 4.0$ \\
MJ21-1 & $7.6 \pm 1.1$ \\
MJ15-132 & $11.4 \pm 1.0$ \\
MJ15-128 & $11.0 \pm 0.6$ \\
MJ15-24a & $10.8 \pm 0.7$ \\
\hline
\end{tabular}

${ }^{\mathrm{z}}$ PME activity of each transgenic line is expressed as relative percentage of PME activity of the WT plants by considering the halo produced by the protein extract of the WT plants as 100\% of PME activity. Data represent the average \pm standard errors of four replicates by using $10 \mu \mathrm{g}$ of total protein extract. were selected for the subsequent analyses. No significant difference in morphology and growth was observed between PMEI transformed lines, lines that had lost the transgene by segregation (hereafter called null segregant [NS]) (MJ1557NS, MJ15-69NS, and MJ15-151NS lines), and the untransformed plants (T. durum Svevo).

Genomic DNA blots of the selected lines were performed using the Acpmei coding region as probe. Digestion with BamHI restriction enzyme, which causes the excision of the Acpmei coding region from the pUbi::Acpmei construct (Fig. 1A), revealed the expected hybridization signal of approximately 500 bp in all transgenic lines (Fig. 1B). Digestion with SphI restriction enzyme, which cuts once within the pUbi:: Acpmei construct, produced a 5.2-kb hybridization fragment in all the transgenic lines that corresponds to the entire construct. This indicates the occurrence of at least two insertions of the cassette as direct tandem repeat into one locus, which is a common result with standard biolistic transformation (Fig. 1C). Additional hybridizing fragments were present in all three transgenic lines, indicating different insertion sites and transgene rearrangement events. In particular, MJ15-151 and MJ1569 showed the higher and lower number, respectively, of additional hybridizing fragments. As expected, no hybridization signals were present on genomic DNA of WT and NS plants (Fig. 1C).

\section{Transcript and protein accumulation of AcPMEI in transgenic wheat lines.}

The Acpmei transcript level in line MJ15-69 and MJ15-151 was six- and fourfold higher, respectively, than in line MJ1557 (Fig. 2A). Correspondingly, the MJ15-69 and MJ15-151

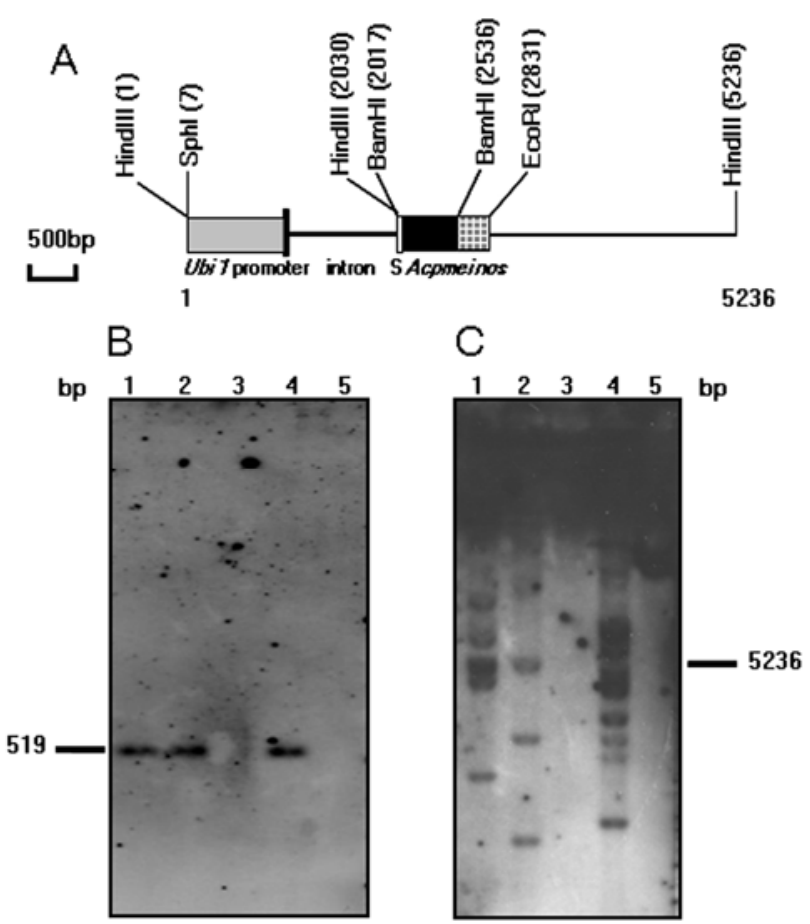

Fig. 1. Schematic representation of the pUbi::Acpmei construct and Southern blots of regenerated transgenic lines. A, The pUbi::Acpmei construct was prepared by cloning the Acpmei gene into the Bam HI site of pAHC17 under control of the maize Ubiquitin1 promoter and NOS terminator. The leader sequence of Pvpgipl (S) has been also added to the mature coding region of Acpmei, for the apoplastic secretion. Genomic DNA (8 $\mu \mathrm{g})$ of $\mathrm{T}_{3}$ transgenic lines was digested with $\mathbf{B}, B a m \mathrm{HI}$ and $\mathbf{C}, S p h \mathrm{I}$ and probed with a digoxigenin-labeled coding region of Acpmei. Lane 1, MJ15-57; lane 2, MJ15-69; lane 3, Triticum durum 'Svevo' (untransformed control); lane 4, MJ15-151; lane 5, null segregant of MJ15-151. 
lines exhibited higher levels of AcPMEI as shown by immunoblotting analysis by using a polyclonal antibody antiAcPMEI (Fig. 2B). These data correlate with the reduced level of PME activity detected in these lines (Table 1).

The same levels of AcPMEI accumulation and PME activity found in leaf extract of the three transgenic lines were also detected in all the other tissues tested, including the spike tissue, a target tissue for some pathogens such as $F$. graminearum, indicating, as expected, that the Ubil promoter regulates a constitutive expression of Acpmei and no tissue-specific degrading activity toward AcPMEI is activated in these tissues (Supplementary Table S1).

Because the pUbi::Acpmei construct contains the entire coding sequence of Acpmei fused to the signal peptide of polyga-
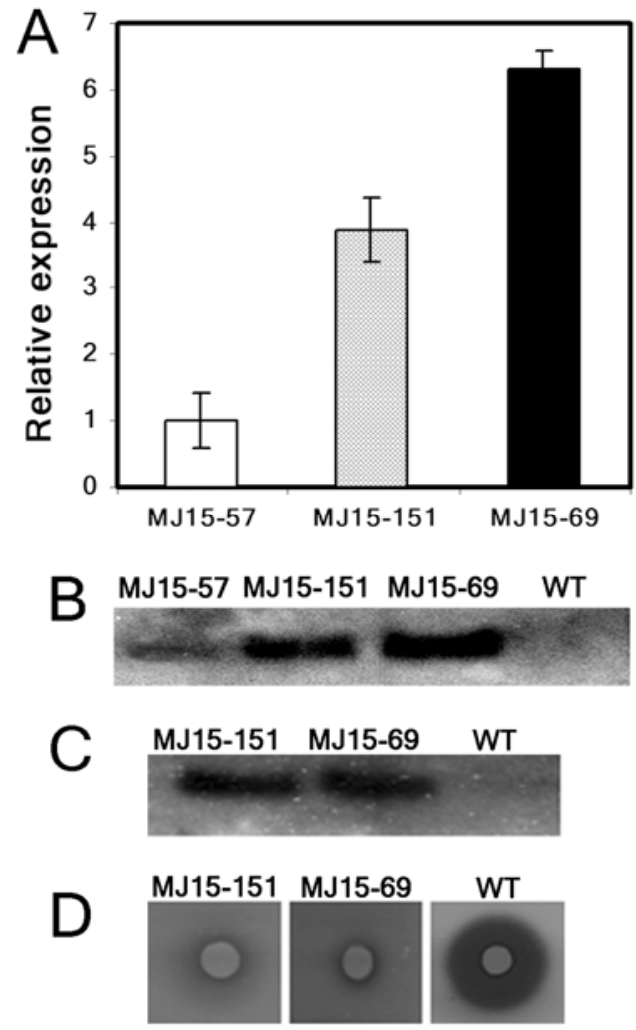

Fig. 2. Expression and activity of pectin methyl esterase inhibitor from Actinidia chinensis (AcPMEI) in the wheat transgenic plants. A, Level of Acpmei transcript in wild-type (WT) and wheat transgenic plants determined by quantitative reverse-transcription polymerase chain reaction. The fold change was relative to the MJ15-57 sample. Bars represent the average \pm standard deviation of three replicates. This experiment was repeated twice with similar results. B, Western blotting of total protein extracts (10 $\mu \mathrm{g}$ ) by using a polyclonal $\mathrm{Ab}$ anti-AcPMEI. C, Western blot of extracellular fluids using a polyclonal Ab anti-AcPMEI. D, Radial gel diffusion assay for pectin methyl esterase (PME) activity of extracellular fluids. The absence of the halo indicates the lack of PME activity. WT, Triticum durum 'Svevo'.

Table 2. Quantification of uronic acids content in the cell walls of Acpmei lines, the null segregant (NS) line, and wild type (WT) plants ${ }^{\mathrm{z}}$

\begin{tabular}{lc}
\hline Lines & Uronic acids $(\boldsymbol{\mu m o l} / \mathbf{m g}$ of cell wall $)$ \\
\hline WT & $0.11 \pm 0.007 \mathrm{a}$ \\
MJ15-151 NS & $0.12 \pm 0.004 \mathrm{a}$ \\
MJ15-57 & $0.12 \pm 0.004 \mathrm{a}$ \\
MJ15-151 & $0.12 \pm 0.007 \mathrm{a}$ \\
MJ15-69 & $0.13 \pm 0.004 \mathrm{a}$ \\
\hline
\end{tabular}

${ }^{\mathrm{z}}$ The same letters indicate not significant differences according to analysis of variance followed by Tukey's test $(P<0.05)$. Data represent average \pm standard error of nine biological replicates. lacturonase-inhibiting protein 1 (Pvpgipl) for the apoplastic targeting, we assayed the extracellular fluids (EF) of lines MJ15-69 and MJ15-151 by immunoblot analysis for the presence of the inhibitor. Both lines showed the AcPMEI accumulation in EF (Fig. 2C) as well as a reduced PME activity with respect to the untransformed WT (Fig. 2D), confirming that AcPMEI is correctly targeted into the apoplastic compartment.

Determination of the degree of pectin methyl esterification and immunochemical analysis of cell walls of wheat transgenic lines.

The degree of pectin methyl esterification (DM) was determined in cell walls extracted from selected Acpmei, WT, and MJ15-151NS lines. All these lines showed a similar content of uronic acids (Table 2). Transgenic lines MJ15-151 and MJ1569 showed a significant increase of DM of approximately 20 and 32\%, respectively (Fig. 3). In contrast, the MJ15-57 line expressing a low level of AcPMEI inhibitor and the MJ15151 NS line showed no significant differences in their DM compared with WT plants (Fig. 3).

In order to investigate the spatial distribution of methyl esters in $\mathrm{HG}$, an immunochemical analysis was performed on chelating agent-soluble solid (ChASS) fractions, which contain mainly pectin, extracted from cell walls of transgenic (MJ15-151 and MJ15-69) and control plants (WT and NS). The analysis was carried out by using monoclonal antibodies that recognize pectin epitopes with different methyl ester distribution. In particular, LM7 and JIM5, which recognize epitopes of HG composed by three or four contiguous unesterified GalA with adjacent or flanking methyl-esterified residues (Clausen et al. 2003; Willats et al. 2001b) and PAM1, which is specific to blockwise de-methyl-esterified HG composed by approximately 30 contiguous GalA residues, were used (Willats et al. 1999, 2001b). Immunodot analysis on cell wall pectin of the transgenic and control plants revealed a lower level of PAM1-binding epitope on both Acpmei lines compared with the WT, indicating a reduced level of long stretches of de-esterified pectin in the transgenic lines (Fig. 4). A reduced level of both JIM5- and LM7-binding epitopes was also detected on both transgenic lines compared with the WT, indicating a low level of short contiguous unesterified GalA residues in the transgenic lines (Fig. 4).

Transgenic wheat lines show increased resistance to $B$. sorokiniana and $\boldsymbol{F}$. graminearum infection.

The resistance to $B$. sorokiniana was analyzed on the first emerged leaf of Acpmei lines MJ15-151, MJ15-69, and MJ1557; their corresponding NS lines MJ15-151NS and MJ15-

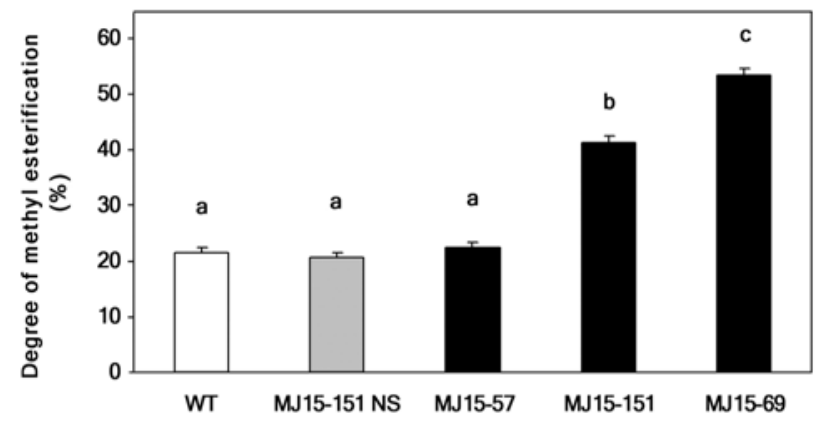

Fig. 3. Determination of the degree of methyl esterification of cell wall pectin from Acpmei lines (MJ15-151, MJ15-69, and MJ15-57), a null segregant line (MJ15-151 NS), and Triticum durum 'Svevo' (WT). Different letters indicate data sets significantly different according to Tukey's test $(P$ $<0.05)$. Values represent the average \pm standard error of three independent experiments with three plants each. 
69NS; and WT plants (T. durum Svevo). Disease symptoms were visible $48 \mathrm{~h}$ postinfection (hpi) and appeared as reddishbrown spots of variable size on the leaf surface. In order to facilitate the analysis of single lesions, data were collected 72 hpi. Disease symptoms were evaluated as the ratio between leaf area showing symptoms and the total leaf area, expressed as percentage. MJ15-151 and MJ15-69 lines show a significant reduction in disease symptoms compared with the control plants. The reduction in symptom severity for both Acpmei lines was higher than $55 \%$ when compared with the corresponding NS lines or WT plants (Fig. 5). In contrast, the MJ15-57 line that expresses a low level of AcPMEI inhibitor showed no significant difference in disease symptoms compared with the control lines (Fig. 5).

Flowering spikes of MJ15-151 and MJ15-69 lines were also subjected to infection with the fungal pathogen $F$. graminearum. Two independent tests were performed by point inoculation of the opposite central spikelets in primary spikes (Zadoks stage 68) of transgenic and control plants (lines MJ15-151NS and MJ15-69NS and WT plants). FHB disease symptoms, manifested as spikelet bleaching, usually appeared 3 days postinfection (dpi) and the spread of the disease was visually examined for a period of 18 days (Fig. 6). Transgenic lines MJ15-69 and MJ15-151 showed a significant reduction of symptoms starting from 9 to 18 dpi compared with NS and WT plants. In particular, at $9 \mathrm{dpi}$, the reduction of FHB symptoms of both MJ15-69 and MJ15-151 was approximately $45 \%$ compared with control plants whereas, at $18 \mathrm{dpi}$, the reduction of symptoms of both MJ15-69 and MJ15-151 was approximately $20 \%$ compared with control plants (Fig. 6).

\section{Pectin methyl esterification affects growth and endopolygalacturonase (PG) activity of $B$. sorokiniana and $F$. graminearum.}

In order to verify whether the reduced FHB and spot blotch symptoms observed on the Acpmei lines were related to the impaired ability of the fungal pathogens to grow on methylesterified pectins, both $B$. sorokiniana and $F$. graminearum were grown on a solid medium containing polygalacturonic acid (PGA) or pectin from apple with 70 to $75 \%$ degree of methyl esterification as sole carbon source. Both fungi showed a reduced growth on highly methylated pectin compared with growth on media containing PGA, and this difference was particularly evident during the initial or middle phase of fungal growth. F. graminearum showed approximately $25 \%$ of growth reduction on methylated pectin (mycelium front distal from the inoculum [in centimeters] \pm standard error: $2.33 \pm 0.10$ on PGA and $1.71 \pm 0.10$ on methylated pectin; $P<0.01)$. Similarly, B. sorokiniana grew better on PGA $(2.27 \pm 0.06)$ than on methylated pectin $(1.99 \pm 0.03)$ with a significant $(P<0.01)$

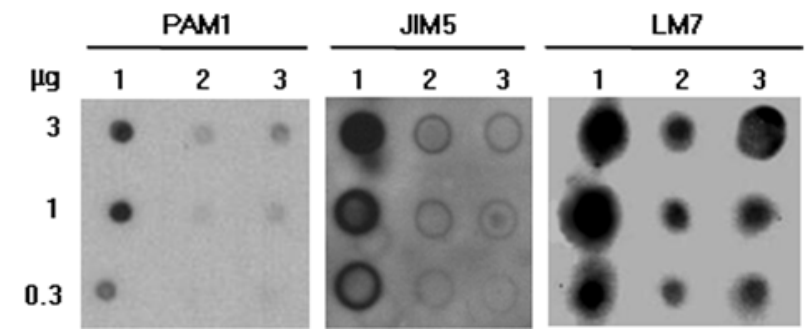

Fig. 4. Immunodot analysis of pectin in Acpmei and wild-type (WT) plants using PAM1, JIM5, and LM7 monoclonal antibodies. The indicated amounts of chelating agent-soluble solid fraction of the different genotypes were applied to a nitrocellulose membrane at corresponding positions for comparison. Lane 1, Triticum durum 'Svevo' (WT); lane 2, MJ15-69; lane 3, MJ15-151. reduction of approximately $12 \%$ on methylated pectin. These results indicate that these fungal pathogens prefer unesterified pectin as carbon source.

To determine whether the cell wall composition of transformed plants affects $F$. graminearum and $B$. sorokiniana growth, we measured the growth on solid medium containing cell walls isolated from WT or MJ15-69 as sole carbon source. A significant reduction of $13 \%(2.05 \pm 0.07$ on WT cell walls and $1.79 \pm 0.03$ on MJ15-69 cell walls; $P<0.01)$ and $10 \%$ (2.22 \pm 0.06 on WT cell walls and $2.00 \pm 0.07$ on MJ15-69 cell walls; $P<0.05$ ) was observed for $F$. graminearum and $B$. sorokiniana growth, respectively, on cell walls of MJ15-69 in comparison with that observed on WT cell walls.

We also verified whether PG activity of $B$. sorokiniana and $F$. graminearum is affected by methyl esterification of the pectic substrate. Agar diffusion assays showed that PG from both fungi have a reduced hydrolytic activity on a highly methylated substrate (70 to $75 \%$ methyl-esterified pectin from apple) compared with that observed on PGA (data not shown). These results were confirmed by also performing a colorimetric reducing end-group assay (Milner and Avigad 1967) on PGA or 70 to $75 \%$ methyl-esterified apple pectin. In particular, the reduction of PG activity on highly methyl-esterified pectin compared with that observed on PGA was 32 and $38 \%$ for the PG from B. sorokiniana and F. graminearum, respectively (Fig. 7). This result indicates that the degree of methylation of the substrate affects PG activity of both $B$. sorokiniana and F. graminearum.

\section{DISCUSSION}

We have produced transgenic wheat plants expressing AcPMEI, a well-characterized pectin methyl esterase inhibitor from kiwi (SwissProt accession number P83326). This inhibitor possesses inhibition activity against a wide range of plant PME (Balestrieri et al. 1990; Camardella et al. 2000; Giovane et al. 2004) and the molecular bases of the interaction with its ligand have been characterized by crystallographic analysis (Di Matteo et al. 2005).

Among the different transgenic lines, we selected the MJ15151 and MJ15-69 lines, showing a high level of AcPMEI, and line MJ15-57, with a lower expression of the transgenic inhibitor. MJ15-151 and MJ15-69 show the strong reduction of endogenous PME activity and a consequently, significantly

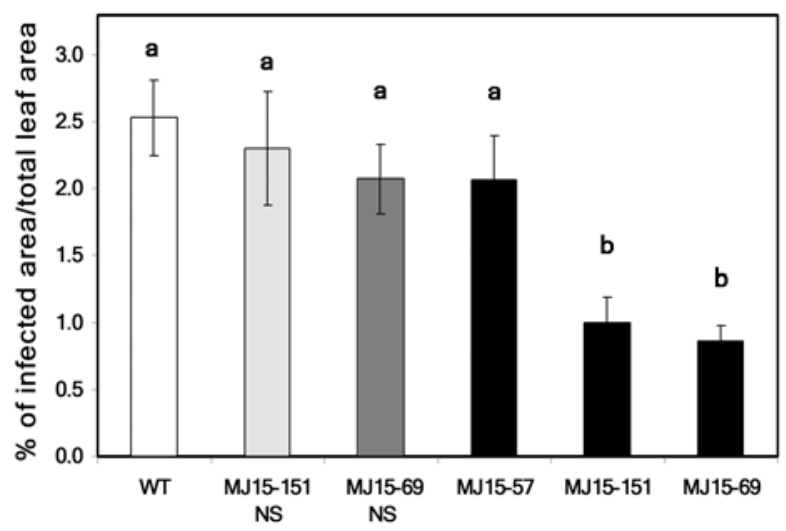

Fig. 5. Quantification of symptomatic leaf area of transgenic plants, null segregant (NS) lines, and wild type (WT) (T. durum 'Svevo') plants inoculated with Bipolaris sorokiniana. Symptom severity (lesion expansion area [square centimeters]/total leaf area [square centimeters]) $\times 100$ at $72 \mathrm{~h}$ postinfection (hpi). Data represent the average \pm standard errors of four experiments performed with at least 15 replicates. Different letters indicate data sets significantly different according to Tukey's test $(P<0.05)$. 
higher DM compared with control plants. In contrast, the MJ15-57 line does not show any significant variation in the DM compared with control plants, indicating that a reduction of PME activity higher than $40 \%$ is required to affect the DM of the cell wall pectin. On the other hand, the total content of uronic acid of wheat Acpmei plants was not significantly different from that of control plants, indicating a similar pectin content and suggesting that the susceptibility of pectin to endogenous hydrolases is not affected.

Levels of de-methyl-esterified pectin of wheat plants expressing AcPMEI were investigated by immunodot assay, using the monoclonal antibody PAM1, which specifically recognizes large de-methyl-esterified blocks of pectin (at least 30 continuous GalA residues) and the antibodies JIM5 and LM7 (which bind pectin with a low degree of methyl esterification). Lines MJ15-151 and MJ15-69, expressing high levels of AcPMEI, showed a lower level of all the three epitopes and, therefore, have a reduced quantity of long stretches of unesterified HG and a higher degree of pectin methyl esterification compared with control plants.

Previous evidence indicates that pectin methyl esterification can play a role in resistance of monocot and dicot plants to bacterial and fungal pathogens (Boudart et al. 1998; Marty et al. 1997; McMillan et al. 1993; Venkatesh 2002; Wydra and Beri 2006). More recently, it was demonstrated that the overexpression of PMEI in Arabidopsis increases the DM of the pectin cell wall and reduces susceptibility of these plants to fungal and bacterial necrotrophs (Lionetti et al. 2007; Raiola et al. 2011). Here, we demonstrated for the first time that the expression of AcPMEI in wheat, a grass species with a low level of pectin in the cell wall, significantly improves the plant resistance to both $B$. sorokiniana and $F$. graminearum. In particular, lines MJ15-151 and MJ15-69, with an increased DM, showed a significant reduction of disease symptoms caused by each of these two fungal pathogens. In contrast, line MJ15-57, with a lower level of expression of AcPMEI and an unmodified DM, showed no improvement in disease resistance.

Plant pathogens produce cell wall-degrading enzymes during infection and, among these, pectinolytic enzymes are the earliest to be secreted (Alghisi and Favaron 1995; Collmer and Keen 1986). Among pectinolytic enzymes, PG play a primary role and, in some plant pathogen interactions, they act as virulence factors. Some examples were previously reported for $\mathrm{Bo}$ - trytis cinerea on tomato (ten Have et al. 1998), Alternaria citri on citrus (Isshiki et al. 2001), Aspergillus flavus on cotton (Shieh et al. 1997), and Claviceps purpurea on rye (Oeser et al. 2002). Both Bipolaris sorokiniana and F. graminearum produce PG during their colonization process of the wheat tissue (Janni et al. 2008; Tomassini et al. 2009), and we demonstrated that the expression of a bean polygalacturonase-inhibiting protein (PvPGIP2) in wheat transgenic plants limits disease symptom development caused by these fungal pathogens (Ferrari et al. 2011; Janni et al. 2008), suggesting that PG play a primary role in these interactions.

In the present article, we showed that highly methyl-esterified pectins are less susceptible to the action of PG of both $B$. sorokiniana and $F$. graminearum, similar to what already was reported for other fungal PG (Bonnin et al. 2002; Kars et al. 2005; Limberg et al. 2000), suggesting that the reduced disease symptoms observed on the Acpmei lines with increased pectin methyl esterification, MJ15-151 and MJ15-69, could be due to the impaired ability of these fungi to colonize host tissue with a high degree of pectin methyl esterification in their cell walls. This possibility is further supported by the evidence of a reduced growth of both fungal pathogens on cell walls isolated from Acpmei plants. Moreover, it has been previously reported that epitopes recognized by LM7 are more sensitive to fungal PG and pectate lyase (Clausen et al. 2003; Willats et al. 2001b). The observation that both MJ15-151 and MJ15-69 show a lower level of LM7 epitopes further indicates that the pectin of these plants has a structure less susceptible to hydrolysis by the PG secreted by the fungus during the infection and colonization of the host tissue.

The Acpmei lines having an increased level of pectin methyl esterification proved to be particularly effective in controlling the spot blotch disease caused by $B$. sorokiniana that reached $50 \%$ at $72 \mathrm{hpi}$, whereas the reduction of FHB symptoms caused by $F$. graminearum was remarkable only at early stages of infection because, at later stages, this effect is attenuated. This reduced resistance of the Acpmei plants in the advanced stage of FHB infection indicates that pectin degradation affects the course of disease mostly at the early stages of $F$. graminearum infection. Later, other virulence factors may become predominant in the spreading of disease. For example, deoxynivalenol, a trichothecene mycotoxin produced by $F$. graminearum during wheat spike infection, is not necessary for initial infec-

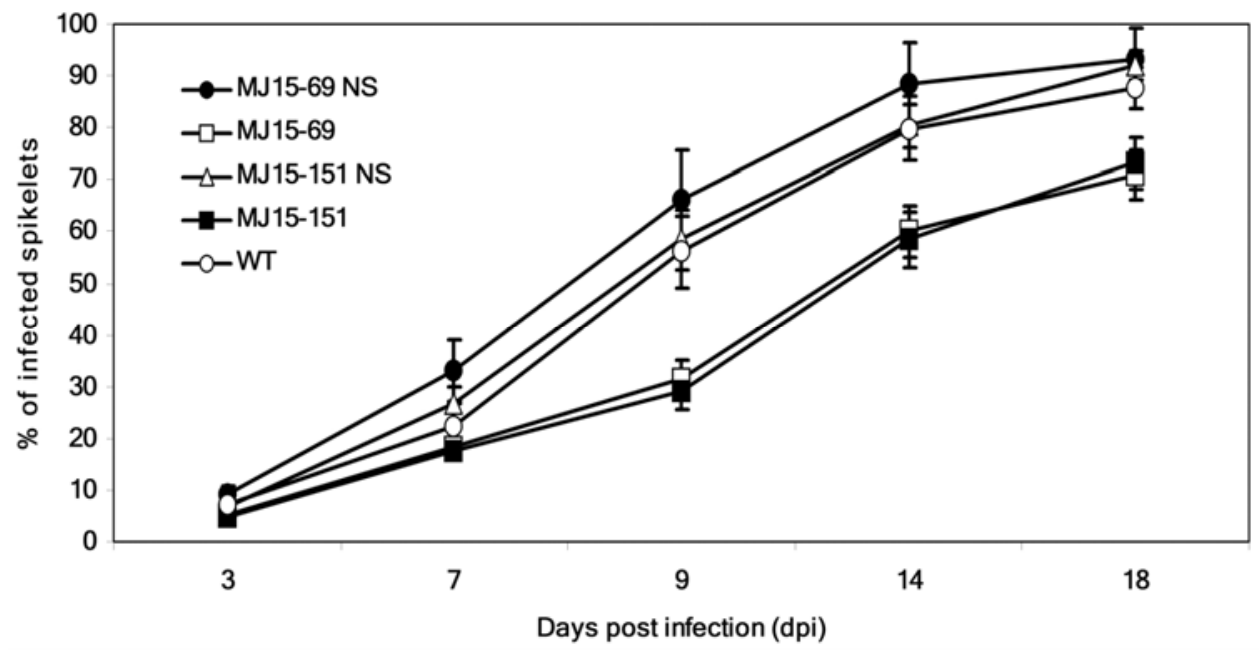

Fig. 6. Time-course analysis of Fusarium head blight symptom development following Fusarium graminearum infection of the transgenic lines MJ15-69 and MJ15-151, the corresponding null segregant lines, and Triticum durum 'Svevo' (wild type [WT]). Disease symptoms are expressed as percentage of spikelets showing symptoms on the total number of spikelets per spike. Data represent the average \pm standard errors of two experiments performed with at least 12 plants per genotype. The average values of lines MJ15-69 and MJ15-151 are significantly different from the corresponding NS lines and WT plants according to Student's $t$ test $(P \leq 0.01)$. 
tion by the fungus but plays a significant role in the spreading of the fungus within a spike (Bai et al. 2001).

The increased resistance of wheat expressing AcPMEI mirrors that reported on Arabidopsis plants overexpressing AtPMEI-1 or AtPMEI-2 against Botrytis cinerea (Lionetti et al. 2007), although the levels of pectin contained in the cell wall of these two plant species are remarkable different (Vogel 2008). This aspect both reinforces the notion of the importance of pectin degradation during wheat colonization by fungal pathogens and highlights the primary role of this polymer for the integrity of the cell wall barrier in monocot plants.

In conclusion, the Acpmei transgenic plants showed a modified methyl esterification of pectin related to a high expression of AcPMEI and a reduced endogenous PME activity. These findings and the observation that the growth rate and PG activity of both $B$. sorokiniana and $F$. graminearum are reduced on highly methyl-esterified pectin and on cell walls of Acpmei plants suggest that the increased resistance of the Acpmei plants to these two pathogens could be due to their altered methyl esterification that reduces the activity of the fungal pectin-degrading enzymes and, in particular, the hydrolytic activity of PG.

\section{MATERIALS AND METHODS}

Generation and characterization of transgenic plants.

To assemble the monocot expression vector pUbi::Acpmei, the mature coding region of Acpmei (accession number P83326), with the leader sequence of the bean polygalacturonase-inhibiting protein 1 (Pvpgip1) (D'Ovidio et al. 2004) for the apoplastic targeting, was inserted into the BamHI site of pAHC17 under control of the maize Ubiquitin 1 promoter and nopaline synthase terminator (Christensen and Quail 1996). The BamHI sites flanking the complete coding region of Acpmei were generated by PCR amplification using the forward and reverse primers (AcpmeiF1Bam) 5'-GAGTGGATC CATGTCTTCAAGCTTAAGCATAA-3' and (AcpmeiR1Bam) 5'-GCGAGGATCCTTAAGAACCTGGAAGAAGAT-3', respectively. PCR reactions were performed in a reaction volume of $25 \mu$ with $10 \mathrm{ng}$ of plasmid DNA (pAcpmei), 2.5 units of FastStart High Fidelity PCR system (Roche Diagnostics, Monza, Italy), 1× Taq PCR buffer, $50 \mathrm{ng}$ of each of the two primers, and $100 \mu \mathrm{M}$ each deoxyribonucleotide. Amplification conditions were 1 cycle at $94^{\circ} \mathrm{C}$ for $2 \mathrm{~min} ; 30$ cycles at $94^{\circ} \mathrm{C}$ for $1 \mathrm{~min}, 60^{\circ} \mathrm{C}$ for $1 \mathrm{~min}$, and $72^{\circ} \mathrm{C}$ for $1 \mathrm{~min}$; and a final step at $72^{\circ} \mathrm{C}$ for $5 \mathrm{~min}$. After $\mathrm{BamHI}$ digestion, the amplicon was inserted into the BamHI site of pAHC17 generating a 5,236-bp plasmid, pUBI::Acpmei. The correct coding sequence of Acpmei and the insertion sites were confirmed by nucleotide sequencing. For the wheat transformation experiments, the plasmid pUbi::bar $(5,505 \mathrm{bp})$, carrying the bar gene that confers resistance to the bialaphos herbicide, was co-bombarded with pUbi::Acpmei $(5,236$ bp) in a 1:3 molar ratio. Constructs were introduced into immature embryos-derived calli of $T$. durum Desf. Svevo as described by Janni and associates (2008). The presence of Acpmei in bialaphos-resistant plants and their progeny was verified by PCR using total DNA obtained from leaf sections of mature plants. DNA amplification was carried out according to the procedures specified for Ready Mix RedTaq polymerase (Sigma-Aldrich, Milan, Italy) at an annealing temperature of $60^{\circ} \mathrm{C}$ by using the specific primers pair AcpmeiF4 (5'-CTTGTATCTTTGAGAACTGCAC) and AcpmeiR4 (5'-TGAGTTGGAATATTTGGTGGAC) that produces an amplicon of $407 \mathrm{bp}$.

To identify homozygotes, a minimum of eight progeny was sampled from each of two successive generations and tested for amplification of Acpmei. Homozygotes of the lines MJ15-
57, MJ15-69, and MJ15-151 were used for further characterization.

DNA extraction and Southern blot analysis were performed as reported in Janni and associates (2008).

Total RNA was extracted from adult plants using the RNasy plant mini kit (Qiagen, Milan, Italy) or the Nucleospin RNA plant kit (Macherey Nagel, Düren, Germany) according to the manufacturer's instructions. Quantitative reverse-transcription (qRT)-PCR experiments were performed using the iCycler (Bio-Rad Laboratories, Monza, Italy) and the master mix iQTMSYBER Green Supermix (BioRad Laboratories) containing the flurogenic SYBER Green I DNA binding dye. Oligonucleotide primer pairs used were AcpmeiF4 (see above) and AcpmeiR2 (5'-CCAAGAGAATCAATAGCATCAGC) for Acpmei and Taact77F (5'-TCCTGTGTTGCTGACTGAGG) and Taact312R (5'-GGTCCAAACGAAGGATAGCA) for Actin (accession number AB181991). The specificity of the primers was verified by nucleotide sequence of the specific amplicon (284 bp for Acpmei gene and 235 bp for the Actin gene). The Actin gene was used as a housekeeping gene.

Total reaction volume was $20 \mu \mathrm{l}$ and included $10 \mu \mathrm{l}(2 \times)$ of Mastermix, $100 \mathrm{ng}$ of cDNA, and $0.5 \mu \mathrm{l}$ of $10 \mu \mathrm{M}$ each forward and reverse primer, and the volume was adjusted with water. Each reaction was made in triplicate. Reaction conditions were as follows: one cycle at $50^{\circ} \mathrm{C}$ for $2 \mathrm{~min}$ and $95^{\circ} \mathrm{C}$ for $5 \mathrm{~min}$; then, 40 cycles at $95^{\circ} \mathrm{C}$ for $30 \mathrm{~s}, 60^{\circ} \mathrm{C}$ for $40 \mathrm{~s}$, and $72^{\circ} \mathrm{C}$ for $30 \mathrm{~s}$.
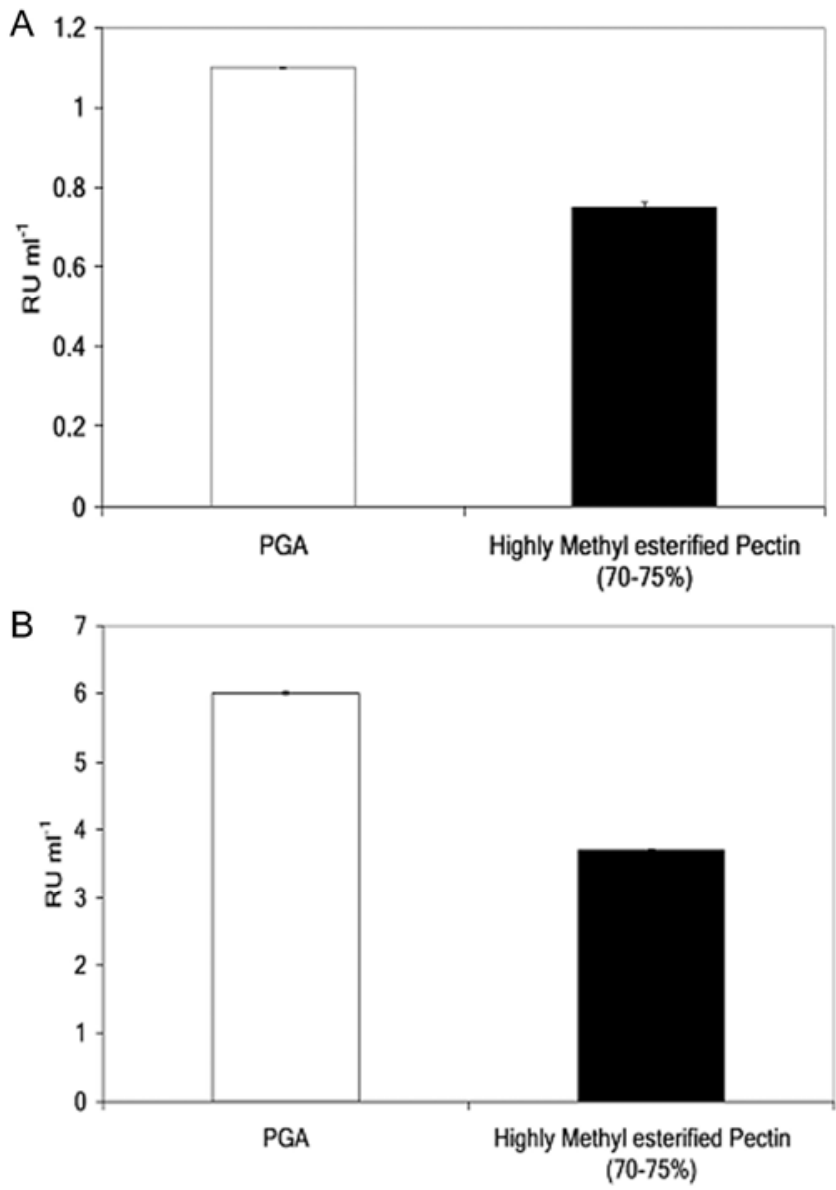

Fig. 7. Endopolygalacturonase (PG) activity of A, Bipolaris sorokiniana and B, Fusarium graminearum on polygalacturonic acid (PGA) or pectin with 70 to $75 \%$ degree of methyl esterification. PG activity is expressed as number of reducing end groups released. Bars represent average \pm standard deviation of three replicates. This experiment was repeated twice with similar results. 
The cycle threshold values of target genes and housekeeping genes were used for further analysis, and samples with more than 0.6 value difference within triplicates were not considered. The relative expression analysis was determined by using the $2^{-\triangle \Delta C T}$ method (Livak and Schmittgen 2001) (Applied Biosystems User Bulletin No. 2-P/N 4303859). Calculation and statistical analyses were performed by Gene Expression Macro Version 1.1 (Bio-Rad Laboratories). The qRT-PCR experiments were performed on RNA samples from two different biological replicas.

Crude protein extracts were obtained by homogenizing wheat tissues in the presence of $20 \mathrm{mM}$ sodium acetate and 1 $\mathrm{M} \mathrm{NaCl}, \mathrm{pH} 4.6(2 \mathrm{ml} / \mathrm{g})$. The homogenate was shaken for $1 \mathrm{~h}$ at $4^{\circ} \mathrm{C}$ and centrifuged for $20 \mathrm{~min}$ at $10.000 \times g$, and the supernatants recovered. Protein concentrations of the crude extracts were determined with the "Bio-Rad Protein assay" kit (BioRad Laboratories). Endogenous PME activity in Acpmei lines in the $T_{1}$ generation and WT plants was quantified by radial gel diffusion assay as reported by Lionetti and associates (2007). Sodium dodecyl sulfate polyacrylamide gel electrophoresis and immunoblot analysis were performed as previously described (Desiderio et al. 1997) using a specific polyclonal antibody raised in rabbit against AcPMEI (Di Matteo et al. 2005).

Extracellular fluids were extracted from wheat seedlings (Zadoks stage 61) by vacuum infiltration with acetate buffer as described by Salvi and associates (1990). The extracellular fluid had negligible glucose-6-phosphate dehydrogenase activity, ruling out cytoplasmic contamination.

Cell walls were isolated from leaves and subsequently analyzed for their degree of methyl esterification and uronic acid content as previously reported by Lionetti and associates (2007).

Immunodot analysis was performed on the ChASS fractions following the procedure reported by Lionetti and associates (2010) using the PAM1ScFv (Willats et al. 1999), LM7, and JIM5 (Clausen et al. 2003) monoclonal antibodies.

PG activity and fungal growth on different pectic substrates.

PG preparations were performed as previously reported by Janni and associates (2008) for Bipolaris sorokiniana (Sacc.) Shoemaker DSMZ 62608 and as described by Tomassini and associates (2009) for F. graminearum Schwabe 3824. PG activity was evaluated using both an agarose diffusion assay (Taylor and Secor 1988) as modified by Ferrari and associates (2003) and by measuring the release of reducing end groups by using the method of Milner and Avigad (1967), as modified by Sella and associates (2004). In both methods, the substrate was either $0.5 \%$ PGA (Sigma-Aldrich) or $0.5 \%$ pectin from apple 70 to $75 \%$ methyl-esterified (Sigma-Aldrich). In the agarose diffusion assay, PG activity was expressed as agarose diffusion units, with one agarose diffusion unit defined as the amount of enzyme that produced a halo of $0.5 \mathrm{~cm}$ in radius (external to the inoculation well of $0.5 \mathrm{~cm}$ in radius) after $18 \mathrm{~h}$ at $30^{\circ} \mathrm{C}$. In the reducing end-groups assay, PG activity was expressed as reducing units (RU). One RU was defined as the amount of enzyme required to release reducing groups at 1 $\mu \mathrm{mol} / \mathrm{min}$ using D-galacturonic acid as standard.

$F$. graminearum and B. sorokiniana were cultured at room temperature on Szécsi solid medium (Szécsi 1990) amended as sole carbon source, with either $1 \%$ (wt/vol) PGA (SigmaAldrich) or $1 \%$ (wt/vol) pectin from apple (Sigma-Aldrich) with 70 to $75 \%$ degree of methyl esterification or $0.3 \%$ (wt/vol) cell walls isolated from transformed (MJ15-69) and WT leaves. Fungal growth was determined as linear expansion by measuring the radius of the mycelium (mycelium front distal from the inoculum) when it reached $50 \%$ of radius expansion. Data from six replicates for each fungus were analyzed statistically applying the Student's $t$ test.

\section{Plant growth and infection assays.}

Wheat seed ( $T$. durum Svevo) were surface sterilized with sodium hypochlorite $(0.5 \% \mathrm{vol} / \mathrm{vol})$ for $20 \mathrm{~min}$ and then rinsed thoroughly in sterile water. Plants were vernalized at $4^{\circ} \mathrm{C}$ for 2 weeks and grown in a climatic chamber at 18 to $23^{\circ} \mathrm{C}$ with a 14-h photoperiod $\left(300 \mu \mathrm{E} \mathrm{m}^{-2} \mathrm{~s}^{-1}\right)$.

For plant inoculation with $F$. graminearum 3824, conidia were recovered from fungal cultures grown on synthetic nutrient agar plates (Urban et al. 2002). Infection experiments of wheat plants were performed by single-spikelet inoculation. A conidia suspension $\left(20 \mu \mathrm{l}, 2.5 \times 10^{4}\right.$ conidia $\left.\mathrm{ml}^{-1}\right)$ supplemented with $0.1 \%$ Tween-20 was pipetted directly through the glumes of two opposite central florets of a wheat head during anthesis. Infected spikes were covered with plastic bags for 2 days in order to maintain high humidity. Disease symptoms were assessed by counting the number of visually diseased spikelets at different dpi and by relating them to the total number of spikelets of the respective head, resulting in a percentage of symptomatic spikelets.

For plant inoculation with B. sorokiniana 62608 DSMZ, conidia were recovered from fungal cultures grown on petri dishes containing potato dextrose agar medium (BD, Sparks, MD, U.S.A.). The infection experiment was carried out following the procedure reported by Janni and associates (2008). Lesion sizes were determined 72 hpi by scanning the leaf surface and calculating the square centimeters of the infected area and of the total area by using the Adobe Photoshop program (Microsoft, Segrate, Italy). The average area per lesion was calculated as weighted arithmetic mean.

Sets of at least 12 plants were infected in each experiment. Each experiment was repeated four times with B. sorokiniana and twice with $F$. graminearum on different batches of plants. $\mathrm{T}_{2}, \mathrm{~T}_{3}$, or $\mathrm{T}_{4}$ progenies were used in the different experiments. Data from both $B$. sorokiniana or $F$. graminearum infection experiments were analyzed statistically applying the Student's $t$ test or by performing analysis of variance followed by Tukey's test.

\section{ACKNOWLEDGMENTS}

Research support for R. D'Ovidio was by the Italian Ministry of University and Research (PRIN 2007 and AgroGen Grants). This work was also supported by the European Research Council (ERC Advanced Grant No. 233083), the Institute Pasteur-Fondazione Cenci Bolognetti, by "Sapienza" University of Rome grant (C26A09RCP9).

\section{LITERATURE CITED}

Alghisi, P., and Favaron, F. 1995. Pectin-degrading enzymes and plantparasite interactions. Eur. J. Plant Pathol. 101:365-375.

Bai, G. H., Desjardins, A. E., and Plattner, R. D. 2001. Deoxivalenolnonproducing Fusarium graminearum causes initial infection, but does not cause disease spread in wheat spikes. Mycopathologia 153:91-98.

Balestrieri, C., Castaldo, D., Giovane, A., Quagliuolo, L., and Servillo, L. 1990. A glycoprotein inhibitor of pectin methylesterase in kiwi fruit (Actinidia chinensis). Eur. J. Biochem. 193:183-187.

Bonnin, E., Le Goff, A., Korner, R., Vigouroux, J., Roepstorff, P., and Thibault, J.-F. 2002. Hydrolysis of pectins with different degrees and patterns of methylation by the endopolygalacturonase of Fusarium moniliforme. Biochim. Biophys Acta 1596:83-94.

Bosch, M., Cheung, A. Y., and Hepler, P. K. 2005. Pectin methylesterase, a regulator of pollen tube growth. Plant Physiol. 138:1334-1346.

Boudart, G., Lafitte, C., Barthe, J. P., Frasez, D., and Esquerre-Tugayé, M. T. 1998. Differential elicitation of defence responses by pectin fragments in bean seedlings. Planta 206:86-94.

Camardella, L., Carratore, V., Ciardiello, M. A., Servillo, L., Balestrieri, C., and Giovane, A. 2000. Kiwi protein inhibitor of pectin methylesterase amino-acid sequence and structural importance of two disulfide bridges. Eur. J. Biochem. 267:4561-4565.

Christensen, A. H., and Quail, P. F. 1996. Ubiquitin promoter-based vec- 
tors for high-level expression of selectable and/or screenable marker genes in monocotyledonous plants. Trans. Res. 5:213-218.

Clausen, M. H., Willats, W. G., and Knox, J. P. 2003. Synthetic methyl hexagalacturonate hapten inhibitors of anti-homogalacturonan monoclonal antibodies LM7, JIM5 and JIM7. Carbohydr Res. 338:17971800

Collmer, A., and Keen, N. T. 1986. The role of pectic enzymes in plant pathogenesis. Annu. Rev. Phytopathol. 24:383-409.

Desiderio, A., Aracri, B., Leckie, F., Mattei, B., Salvi, G., Tigelaar, H., Van Roekel, J. S., Baulcombe, D. C., Melchers, L. S., and De Lorenzo, G. 1997 Polygalacturonase inhibiting proteins (PGIPs) with different specificities are expressed in Phaseolus vulgaris. Mol. Plant-Microbe Interact. 10:852-860.

Di Matteo, A., Giovane, A., Raiola, A., Camardella, L., Bonivento, D., De Lorenzo, G., Cervone, F., Bellincampi, D., and Tsernoglou, D. 2005 Structural basis for the interaction between pectin methylesterase and a specific inhibitor protein. Plant Cell 17:849-858.

D’Ovidio, R., Raiola, A., Capodicasa, C., Devoto, A., Pontiggia, D. Roberti, S., Galletti, R., Conti, E., O’Sullivan, D., and De Lorenzo, G. 2004 Characterization of the complex locus of Phaseolus vulgaris encoding polygalacturonase-inhibiting proteins (PGIPs) reveals subfunctionalization for defense against fungi and insects. Plant Physiol. 135:2424-2435

Ferrari, S., Vairo, D., Ausubel, F. M., Cervone, F., and De Lorenzo, G 2003. Tandemly duplicated Arabidopsis genes that encode polygalacturonase-inhibiting proteins are regulated co-ordinately by different signal transduction pathways in response to fungal infection. Plant Cell 15:93106.

Ferrari, S., Sella, L., Janni, M., De Lorenzo, G., Favaron, F., and D’Ovidio, R. 2011. Transgenic expression of polygalacturonase-inhibiting proteins in Arabidopsis and wheat increases resistance to the flower pathogen Fusarium graminearum. Plant Biol. doi:10.1111/j.1438-8677.2011.00449.x. Published online.

Giovane, A., Servillo, L., Balestrieri, C., Raiola, A., D’Avino, R., Tamburrini, M., Ciardiello, M. A., and Camardella, L. 2004. Pectin methylesterase inhibitor. Biochim. Biophys. Acta 1696:245-252.

Isshiki, A., Akimitsu, K., Yamamoto, M., and Yamamoto, H. 2001. Endopolygalacturonase is essential for citrus black rot caused by Alternaria citri but not brown spot caused by Alternaria alternata. Mol. Plant-Microbe Interact. 14:749-757.

Janni, M., Sella, L., Favaron, F., Blechl, A.E., De Lorenzo, G., and D'Ovidio R. 2008. The expression of a bean pgip in transgenic wheat confers increased resistance to the fungal pathogen Bipolaris sorokiniana. Mol. Plant-Microbe Interact. 21:171-177.

Kars, I., Krooshof, G. H., Wagemakers, L., Joosten, R., Benen, J. A., and van Kan, J. A. 2005. Necrotizing activity of five Botrytis cinerea endopolygalacturonases produced in Pichia pastoris. Plant J. 43:213-225.

Limberg, G., Korner, R., Buchholt, H. C., Christensen, T. M., Roepstorff, P., and Mikkelsen, J. D. 2000. Analysis of different de-esterification mechanisms for pectin by enzymatic fingerprinting using endopectin lyase and endopolygalacturonase II from A. niger. Carbohydr. Res. 327:293-307

Lionetti, V., Raiola, A., Camardella, L., Giovane, A., Obel, N., Pauly, M., Favaron, F., Cervone, F., and Bellincampi, D. 2007. Overexpression of pectin methyl-esterase inhibitors in Arabidopsis restricts fungal infection by Botrytis cinerea. Plant Physiol. 143:1871-1880.

Lionetti, V., Francocci, F., Ferrari, S., Volpi, C., Bellincampi, D., Galletti, R., D’Ovidio, R., De Lorenzo, G., and Cervone, F. 2010. Engineering the cell wall by reducing demethylesterified homogalacturonan improves saccharification of plant tissues for bioconversion. Proc. Natl. Acad. Sci. 107:616-621.

Livak, K. J., and Schmittgen, T. D. 2001. Analysis of relative gene expression data using real-time quantitative PCR and $2^{-\Delta \Delta C T}$ method. Methods 25:402-408

Marty, P., Jouan, B., Bertheau, Y., Vian, B., and Goldberg, R. 1997. Charge density in stem cell walls of Solanum tuberosum genotypes and susceptibility to blackleg. Phytochemistry 44:1435-1441.

McMillan, G. P., Hedley, D., Fyffe, L., and Pérombelon, M. C. M. 1993. Potato resistance to softrot erwinias is related to cell wall pectin esterification. Physiol. Mol. Plant Pathol. 42:279-289.

Milner, Y., and Avigad, G., 1967. A copper reagent for the determination of hexuronic acids and certain ketohexoses. Carbohydr. Res. 4:359-361.

Mohnen, D. 2008. Pectin structure and biosynthesis. Curr. Opin. Plant Biol. 11:266-277.

Oeser, B., Heidrich, P. M., Muller, U., Tudzynski, P., and Tenberge, K. B 2002. Polygalacturonase is a pathogenicity factor in Claviceps purpurea/rye interaction. Fungal Genet. Biol. 36:176-186.

Peaucelle, A., Louvet, R., Johansen, J. N., Hofte, H., Laufs, P., Pelloux, J., and Mouille, G. 2008. Arabidopsis phyllotaxis is controlled by the methyl-esterification status of cell-wall pectins. Curr. Biol. 18:1943-1948.

Pelletier, S., Van Orden, J., Wolf, S., Vissenberg, K., Delacourt, J., Ndong, Y. A., Pelloux, J., Bischoff, V., Urbain, A., Mouille, G., Lemonnier, G., Renou, J.-P., and Höfte, H. 2010. A role for pectin de-methylesterification in a developmentally regulated growth acceleration in dark-grown Arabidopsis hypocotyls. New Phytol. 188:726-739.

Pelloux, J., Rusterucci, C., and Mellerowicz, E. J. 2007. New insights into pectin methyl-esterase structure and function. Trends Plant Sci. 12:267277.

Raiola, A., Camardella, L., Giovane, A., Mattei, B., De Lorenzo, G., Cervone, F., and Bellincampi, D. 2004. Two Arabidopsis thaliana genes encode functional pectin methylesterase inhibitors. FEBS (Fed. Eur. Biochem. Soc.) Lett. 557:199-203.

Raiola, A., Lionetti, V., Elmaghraby, I., Immerzeel, P., Mellerowicz, E. J., Salvi, G., Cervone, F., and Bellincampi, D. 2011. Pectin methylesterase is induced in Arabidopsis upon infection and is necessary for a successful colonization by necrotrophic pathogens. Mol. Plant-Microbe Interact. 24:432-440.

Salvi, G., Giarrizzo, F., De Lorenzo, G., and Cervone, F. 1990. A polygalacturonase-inhibiting protein in the flowers of Phaseolus vulgaris L. J. Plant Physiol. 136:513-518.

Sella, L., Castiglion, C., Roberti, S., D’Ovidio, R., and Favaron, F. 2004. An endo-polygalacturonase (PG) of Fusarium moniliforme escaping inhibition by plant polygalacturonase-inhibiting protein (PGIPs) provides new insights into the PG-PGIP interaction. FEMS (Fed. Eur. Microbiol. Soc.) Microbiol. Lett. 240:117-124.

Shieh, M. T., Brown, R. L., Whitehead, M. P., Cary, J. W., Cotty, P. J. Cleveland, T. E., and Dean, R. A. 1997. Molecular genetic evidence for the involvement of a specific polygalacturonase, $\mathrm{P} 2 \mathrm{c}$, in the invasion and spread of Aspergillus flavus in cotton bolls. Appl. Environ. Microbiol. 63:3548-3552.

Szécsi, A. 1990. Analysis of pectic enzyme zymograms of Fusarium species. II. Comparison of polygalacturonase zymograms of Fusarium culmorum and Fusarium graminearum. J. Phytopathol. 130:188-196.

Taylor, R. J., and Secor, G. A. 1988. An improved diffusion assay for quantifying the polygalacturonase content of Erwinia culture filtrates. Phytopathology 78:1101-1103.

ten Have, A., Mulder, W., Visser, J., and van Kan, J. A. 1998. The endopolygalacturonase gene Bcpg1 is required for full virulence of Botrytis cinerea. Mol. Plant-Microbe Interact. 11:1009-1016.

Tomassini, A., Sella, L., Raiola, A., D’Ovidio, R., and Favaron, F. 2009. Characterization and expression of Fusarium graminearum endo-polygalacturonases in vitro and during wheat infection. Plant Pathol 58:556-564.

Urban, U., Daniels, S., Mott, E., and Hammond-Kosack, K. 2002. Arabidopsis is susceptible to the cereal ear blight fungal pathogens Fusarium graminearum and Fusarium culmorum. Plant J. 32:961-973.

Venkatesh, B. 2002. Characterization of bacterial lipopolysaccharides (Pseudomonas syringae pv. tomato and Pseudomonas syringae pv. apii) and pectins of tomato and celery plants (Lycopersicon esculentum and Apium graveolens) regarding their possible role in host/pathogen interaction. Ph.D. thesis, University of Göttingen, Germany.

Vogel, J. 2008. Unique aspects of the grass cell wall. Curr. Opin. Plant Biol. 11:301-307.

Wietholter, N., Graebner, B., Mierau, M., Mort, A. J., and Moerschbacher, B. M. 2003. Differences in the methyl ester distribution of homogalacturonans from near-isogenic wheat lines resistant and susceptible to the wheat stem rust fungus. Mol. Plant-Microbe Interact. 16:945-952.

Willats, W. G., Gilmartin, P. M., Mikkelsen, J. D., and Knox, J. P. 1999. Cell wall antibodies without immunization: Generation and use of deesterified homogalacturonan block-specific antibodies from a naive phage display library. Plant J. 18:57-65.

Willats, W. G., McCartney, L., Mackie, W., and Knox, J. P. 2001a. Pectin: Cell biology and prospects for functional analysis. Plant Mol. Biol. 47:9-27.

Willats, W. G., Orfila, C., Limberg, G., Buchholt, H. C., Van Alebeek, G. J., Voragen, A. G., Marcus, S. E., Christensen, T. M., Mikkelsen, J. D., Murray, B. S., and Knox, J. P. 2001b. Modulation of the degree and pattern of methyl-esterification of pectic homogalacturonan in plant cell walls. Implications for pectin methyl esterase action, matrix properties, and cell adhesion. J. Biol. Chem. 276:19404-19413.

Wolf, S., Mouille, G., and Pelloux, J. 2009. Homogalacturonan methylesterification and plant development. Mol. Plant 2:851-860.

Wydra, K., and Beri, H. 2006. Structural changes of homogalacturonan, rhamnogalacturonan I and arabinogalactan protein in xylem cell walls of tomato genotypes in reaction to Ralstonia solanacearum. Physiol. Mol. Plant Pathol. 68:41-50. 